\title{
The Effect of Interdiffusion on the Intersubband Optical Properties in a Modulation-Doped Quantum-Well Structure
}

\author{
E. Herbert Li, Senior Member, IEEE
}

\begin{abstract}
The linear and nonlinear (based on optical field intensity) intersubband absorptions in conduction band, and its change in refractive index in AlGaAs-GaAs interdiffused quantum wells (QW's) are presented. The calculation of the electron energy levels and the envelope wave functions in a modulation doped interdiffused QW's with screening effects are considered. QW interdiffusion shows a wavelength tunability of the intersubband absorption peaks and refractive index dispersions. This shifting of the transition energies is also demonstrated here to be a useful technique for broad-band and multicolor photodetector application. In addition, it can serve to remove noise, such as minor peaks and dispersions, in the optical spectra.
\end{abstract}

Index Terms - Infrared detectors, modulation doping, nonlinear optics, quantum-well interdiffusion, quantum-well intermixing.

\section{INTRODUCTION}

$\mathbf{S}$ EMICONDUCTOR quantum-well (QW) heterostructures have attracted a great deal of attention because of their novel electronic and optical properties over the past two decades. The confinement of carriers in the semiconductor QW's and superlattice structures, which lead to the quantization of energy levels and the formation of subbands, have been studied extensively [1]. These energy levels can be tailored by changing the barrier widths and heights, well widths, and by modifying the shape of QW and external applied electric fields. The use of nonsquare shaped QW's with a nonlinear graded bandgap profile along the growth direction, such as those produced by interdiffusion, are of growing interest for the controlled modification of the properties of QW structures [2]. This process modifies the quantized energy levels and the respective wave functions of the as-grown $\mathrm{QW}$ structure [3]. Consequently, the optical properties of the diffused QW (DFQW) are modified and can be used to develop novel optoelectronic devices [4].

The first observation of the intersubband transition between the ground and the excited states of modulation doped GaAs QW's by West and Eglash [5] in the infrared (IR) region has stimulated a growing interest in mid-IR wavelength applications [6]-[8]. These include nonlinear optical rectification

Manuscript received August 21, 1996; revised February 10, 1998. This work was supported by the Hong Kong Research Grant Council earmarked grant.

The author was with the Division of Applied Sciences, Harvard University, Cambridge, MA 02138 USA, on leave from the Department of Electrical \& Electronic Engineering, University of Hong Kong, Hong Kong.

Publisher Item Identifier S 0018-9197(98)04563-1.
[9] and carrier-induced effects [10]. In addition, intersubband transitions in DFQW has been investigated and demonstrated for wavelength tuning in the $8-12-\mu \mathrm{m}$ range in the case of multiple QW's [11] and in 12-60- $\mu \mathrm{m}$ range in the case of single QW's [12]. Postgrowth wavelength tuning of QW infrared detectors by rapid thermal annealing has also been realized [13]-[15]. They are very useful for IR imaging and atmospheric sensing. Theoretical calculations of linear and nonlinear intersubband optical transitions in a QW have been discussed [16]-[19]. The intersubband absorption for modulation-doped square QW's have also been investigated [20]. However, the effects of interdiffusion on the modulation doped QW structures have not yet been analyzed. This is the first time such investigation is performed on such structures.

Carrier effects on interdiffused modulation-doped semiconductor square QW structures have been studied [21], [22]. The spatial separation between the charged dopant atoms and the free electronic carriers creates an electric potential in a modulation-doped QW structure. This induced electric potential will modify the original potential profile which includes the built-in and the external applied potentials. The modification of the overall potential profile leads to the changes in electron energy levels and envelope wave functions. It further leads to changes in the electronic and optical properties of the QW.

In this paper, the intersubband optical processes in a modulation doped AlGaAs-GaAs DFQW are considered. In order to determine the influence of interdiffusion and the carrier effects on the QW thoroughly, DFQW's with different well widths and aluminum concentrations are considered in several diffusion extents. The carrier-induced effects are taken into account by solving the Schrödinger and Poisson's equations self-consistently. The transition energies, linear absorption coefficients, and refractive index changes in these modulationdoped DFQW's are analyzed for potential applications in midto far-IR photodetection. In addition, the nonlinear effect due to the optical intensity is examined and the saturation intensity is also considered. In Section II, formulation of the linear and nonlinear intersubband optical-absorption coefficients and refractive indices in a DFQW is presented and the procedures for solving the consistent Schrödinger equation and Possion equations are discussed. In Section III, the eigenstate properties and the nonlinear properties due to optical field intensity and carrier effect are analyzed. Furthermore, optimization based on the application of interdiffusion to the intersubband absorption and change of refractive index in DFQW's are 
investigated. A wide bandwidth material for the applications of a multicolor photodetector and a broad-band material for photodetection are also proposed. Finally, the conclusions are given in Section IV.

\section{Modeling OF A Modulation-Doped DFQW}

\section{A. Intersubband States}

Modulation-doped AlGaAs-GaAs interdiffused single QW structures are discussed in this paper. The diffused Al composition profile $w(z)$ across the $\mathrm{QW}$ structure is given by

$$
w(z)=w_{o}\left\{1-\frac{1}{2}\left[\operatorname{erf}\left(\frac{L_{z}+2 z}{4 L_{d}}\right)+\operatorname{erf}\left(\frac{L_{z}-2 z}{4 L_{d}}\right)\right]\right\}
$$

where $w_{o}$ is the as-grown $\mathrm{Al}$ mole fraction in the barrier, $L_{z}$ is the as-grown width of the $\mathrm{QW}, z$ is both the quantization and the grown axis (QW centered at $z=0)$, and erf denotes the error function. The various stages of interdiffusion are characterized by the diffusion length $\left[L_{d}=(D t)^{1 / 2}\right.$ where $D$ and $t$ are the diffusion coefficient and annealing time, respectively]. A small value of $L_{d}$ corresponds to a slightly interdiffused QW with the compositional profile almost rectangular and a large value of $L_{d}$ corresponds to a more extensively interdiffused $\mathrm{QW}$ structure. The compositional profile in the QW structure after interdiffusion implies that the carrier effective mass and the bulk bandgap vary continuously across the QW. The interdiffusion-induced DFQW conduction band confinement profile $V_{C}(z)$ is defined here by

$$
V_{C}(z)=Q_{C}\left[E_{g}(z)-E_{g}(z=0)\right]
$$

where the zero potential is defined at the bottom $(z=0)$ of the interdiffused QW and positively up in the conduction band, the absolute position of this zero potential (with respect to the bottom of the DFQW) which varies with $L_{d}$, and $Q_{C}$ is the band offset splitting ratio.

Using the Ben-Daniel and Duke model [23] with a $z$ position-dependent effective mass on the interdiffused composition profile, by the one-dimensional (1-D) Schrödinger-like equation

$$
-\frac{\hbar^{2}}{2} \frac{d}{d z}\left[\frac{1}{m_{e}^{*}(z)} \frac{d \psi_{r \ell}(z)}{d z}\right]+V_{C}(z) \psi_{\ell}(z)=E_{\ell} \psi_{\ell}(z)
$$

where $\ell=1,2, \cdots$ are the QW subband levels for the electrons, $m_{e}^{*}[w(z)]$ is the electron effective mass in the $z$ direction. Equation (3) is solved numerically using a finitedifference method with the confinement profile defined in (2) and the boundary condition is taken to be zero at the end of the barrier [24], and the eigenstates can now be obtained.

In the DFQW considered here, the electrons, heavy holes, and light holes in each subband are considered as quasi-twodimensional. For the $\ell$ th subband, the surface concentration of carriers $N_{r \ell}\left(\mathrm{cm}^{-2}\right)$ can be expressed as

$$
N_{r \ell}=\left(\frac{m_{r}^{*}}{\pi \hbar^{2}}\right) k_{B} T \ln \left\{1+\exp \left[\frac{\left(E_{F}-E_{\ell}\right)}{k_{B} T}\right]\right\}
$$

where $m_{r}^{*}$ is the effective mass of the electron, heavy hole, and light hole inside the QW for $r=e, h h$, and $l h$, respectively. $w$ is the $\mathrm{Al}$ concentration ratio inside the $\mathrm{QW}$ and $m_{\circ}$ is the free electron mass. $E_{F}$ is the Fermi level energy of the QW and $E_{\ell}$ is the energy of each subband where the subscript $\ell$ refers to the number of that subband. $T$ is the temperature in degrees Kelvin and $k_{b}$ is the Boltzmann's constant. The total density of carriers $N_{r s}$ in the DFQW is summing over all subbands in (4), i.e.,

$$
N_{r s}=\sum N_{r \ell}
$$

The total surface concentration $N_{r s}$ is equal to the doping concentration times the total width of the doped region. The density of carriers $n_{r s}$ in the GaAs well are obtained by dividing surface concentration $N_{r s}$ by the well width. The quasi-Fermi level can then be obtained by solving (4) and (5) with the Schrödinger equation and Poisson's equation self-consistently [21]

$$
\begin{aligned}
\frac{d^{2}}{d z^{2}} V_{H e}(z) & =-\frac{4 \pi e}{\varepsilon}\left[n_{h h}+n_{l h}-n_{e}(z)\right] \\
V_{H h h}(z) & =V_{H l h}(z) \\
& =-V_{H e}(z)
\end{aligned}
$$

where $n_{e}, n_{h h}, n_{l h}$ are the spatial distributions for electron, heavy hole, and light hole, respectively. The Hartree terms $V_{H e}, V_{H h h}$, and $V_{H l h}$ are obtained from the Poisson equation, and the boundary condition has taken these Hartree terms equal to zero at $z= \pm 50 \mathrm{~nm}$. The $\mathrm{QW}$ envelope wave functions (eigenstates) will finally be obtained until the solutions of (3) and (6) converge. Since the envelope wave functions are slowly varying compared with those for the Bloch wave functions, the matrix elements can be written approximately as [25]

$$
M_{\ell \ell^{\prime}}=|q| \int_{-\infty}^{+\infty} \Psi_{\ell}(z) z \Psi_{\ell^{\prime}}(z) d z
$$

where $q$ is the electronic charge, the subscript $\ell$ refers to the initial state, and $\ell^{\prime}$ refers to the final state.

\section{B. Optical Properties}

With the Fermi level and the envelope wave functions calculated, the linear and nonlinear intersubband absorption coefficients and the changes in refractive indices to the optical radiation in the conduction band are readily obtained [21]

$$
\begin{aligned}
\alpha(\omega)= & \omega \sqrt{\frac{\mu}{\varepsilon}} \operatorname{Im}\left[\varepsilon_{o} \chi_{z z}(\omega)\right] \\
= & \omega \sqrt{\frac{\mu}{\varepsilon}} \sum_{\ell^{\prime}} \sum_{\ell} \frac{\left|M_{\ell^{\prime}}^{z}\right|^{2}\left(n_{\ell}-n_{\ell^{\prime}}\right)(\hbar \gamma)}{\left(E_{\ell^{\prime}}-E_{\ell}-\hbar \omega\right)^{2}+(\hbar \gamma)^{2}} \\
& \cdot\left(\frac{1}{1+\frac{I_{\mathrm{op}}}{I_{\mathrm{op}}^{s}}}\right)
\end{aligned}
$$




$$
\begin{aligned}
\Delta n_{r}(\omega)= & \sqrt{\frac{\varepsilon}{\varepsilon_{o}}}\left(\frac{1}{2 \varepsilon}\right) \operatorname{Re}\left[\varepsilon_{o} \chi_{z z}(\omega)\right] \\
= & \left(\frac{1}{2 n_{r} \varepsilon_{o}}\right) \operatorname{Re}\left[\varepsilon_{o} \chi_{z z}(\omega)\right] \\
= & \left(\frac{1}{2 n_{r} \varepsilon_{o}}\right) \\
& \cdot \sum_{\ell^{\prime}} \sum_{\ell} \frac{\left|M_{\ell^{\prime}}^{z}\right|^{2}\left(n_{\ell^{\prime}}-n_{\ell}\right)\left(E_{\ell^{\prime}}-E_{\ell}-\hbar \omega\right)}{\left(E_{\ell^{\prime}}-E_{\ell}-\hbar \omega\right)^{2}+(\hbar \gamma)^{2}} \\
& \cdot\left(\frac{1}{1+\frac{I_{\mathrm{op}}}{I_{\mathrm{op}}^{s}}}\right)
\end{aligned}
$$

where

$$
\begin{aligned}
I_{\mathrm{op}} & =2 n_{r}|E|^{2} / \mu c, \\
I_{\mathrm{op}}^{s} & =\frac{n_{r}}{2 \mu c} \frac{\left(E_{\ell^{\prime}}-E_{\ell}-\hbar \omega\right)^{2}+(\hbar \gamma)^{2}}{\left|M_{\ell \ell^{\prime}}^{z}\right|^{2}} . \\
n_{\ell} & =\frac{2}{V} \sum_{k_{t}} \rho_{\ell \ell}^{(0)}
\end{aligned}
$$

and

$$
n_{\ell^{\prime}}=\frac{2}{V} \sum_{k_{t}} \rho_{\ell^{\prime} \ell^{\prime}}^{(0)}
$$

where $n_{\ell}$ and $n_{\ell^{\prime}}$ are the densities of electrons in the initial $(\ell)$ and the final $\left(\ell^{\prime}\right)$ subbands of the intersubband transition of the unperturbed system, $M^{z}$ is the dipole operator in the direction of the $\mathrm{QW}, k_{t}$ is the wave vector in the $x-y$ plane, and $\rho^{(0)}$ is the single-electron-density operator for the unperturbed system. $c$ is the speed of light, $\gamma, \mu, \varepsilon$, and $n_{r}$ are the relaxation time, permeability, real part of permitivitty, and the refractive index of the DFQW, respectively. $I_{\mathrm{op}}$ is the intensities of incident optical radiation and $I_{\mathrm{op}}^{s}$ is the saturation intensity of the intersubband optical transition between two subbands $\ell$ and $\ell^{\prime}$, respectively, and $E$ is the electric field of the optical radiation.

\section{RESULTS AND DISCUSSION}

In this section, theoretical calculations on the electron energy levels, linear and nonlinear intersubband absorption coefficients, and changes in refractive indices of the DFQW conduction band for various DFQW structures, carrier injection levels, and diffusion lengths are analyzed.

The modulation-doped AlGaAs-GaAs QW structure is considered. The as-grown aluminum concentration in the barrier is set at $w=0.2,0.3$, and 0.4, and well width equals $L_{z}=70$, 90, and $130 \AA$. The doping concentration of $\mathrm{Si}$ at a distance $200 \AA$ away from the well center includes $10^{16}, 10^{17}$, and $10^{18}$ $\mathrm{cm}^{-3}$. Finally, the diffusion length, which represents the extent of interdiffusion, is varied from $L_{d}=0$ to $40 \AA$. These ranges of the structural parameters represent QW's with different shapes, such as tall and narrow or short and wide and with a continuously varying graded profile at three (high to low) carrier injection levels. This permits a more comprehensive analysis of the DFQW and also allows optimization of the QW
TABLE I

List of Parameters. $w$ is the Al Concentration Ratio, $m_{o}$ is the Free Electron Mass, and $\varepsilon_{o}$ is the Dielectric Constant of Free Space

\begin{tabular}{ccc}
\hline & Units & $\mathrm{Al}_{w} \mathrm{Ga}_{1-w} \mathrm{As}$ \\
\hline $\mathrm{E}_{g}(\mathrm{eV})$ & $\mathrm{eV}$ & $1.424+1.594 w+w(1-w)(0.127-$ \\
$m_{e}$ & $m_{o}$ & $1.31 w)$ \\
$\epsilon$ & $\epsilon_{0}$ & $0.0632+0.856 w+0.0231 w^{2}$ \\
$T$ & $\mathrm{~K}$ & $13.18-3.12 w$ \\
$\hbar \gamma$ & $\mathrm{meV}$ & 500 \\
$I_{\mathrm{op}}$ & $\mathrm{MW} / \mathrm{cm}^{\wedge} 2$ & 5 \\
$\mu$ & $\mu_{\circ}$ & 1 \\
\hline
\end{tabular}
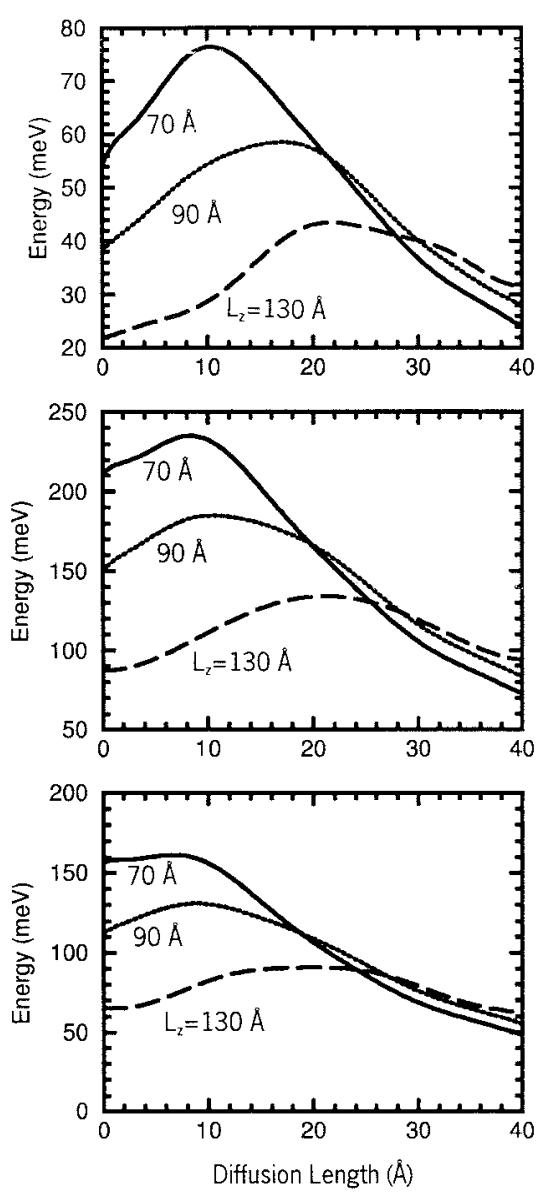

Fig. 1. (a) Ground state energy $E_{1}$ against diffusion length, (b) first excited state energy $E_{2}$ against diffusion length, and (c) intersubband transition energy $\Delta E_{12}=E_{2}-E_{1}$ against diffusion length. The as-grown $\mathrm{QW}$ has $\mathrm{Al}$ concentration $=0.3$ and doping concentration $=10^{17} \mathrm{~cm}^{-3}$. Various cases of as-grown width $L_{z}$ are shown.

to be found. The QW material parameters used for calculation are listed in Table I.

\section{A. Intersubband Transition}

The DFQW structures contain at most five eigenstates for all cases considered in this paper. Fig. 1 shows the ground state energy $E_{1}$, first excited state $E_{2}$, and their difference as a function of diffusion length up to $40 \AA$ for cases of $L_{z}=$ 70,90 , and $130 \AA$, with doping concentration equal to $10^{17}$ $\mathrm{cm}^{-3}$. Since the wavelength position of the peak intersubband 
absorption is mainly contributed by the intersubband transition energy between the ground state and the first excited state, therefore, in order to analyze the trend for the shifting of wavelengths as a function of $L_{d}$, it is necessary to know how the subband eigenenergies vary.

As interdiffusion proceeds, both $E_{1}$ and $E_{2}$ energies rise initially to a maximum and then decrease monotonically toward zero as QW intermixing becomes more complete. This maximum appears earlier for narrower QW's. The reason for the rise is caused by a narrowing well as a consequence of interdiffusion and is well documented [26]. The narrowing of the QW process is more rapid for narrower as-grown well widths. The reason for a delayed energy peak in the case of a wider well width is due to the diffusing atoms require a longer time (hence longer $L_{d}$ ) to reach the center of the well from the heterointerface.

The range of variation of the second subband is much larger than the first subband, especially in a QW with smaller well widths (e.g., $L_{z}<90 \AA$ ). As a consequence, the variation of the transition energies between the first two subbands are also affected as interdiffusion proceeds. Since the intersubband absorption is mainly contributed from the first two subbands, the transition energy between them are very important for choosing the operation wavelength for the photodetector and modulator. Fig. 1(c) shows the intersubband transition energy, $\Delta E_{12}=E_{2}-E_{1}$. As can be seen, $\Delta E_{12}$ increases (blue shift) initially for all well widths and eventually decreases (red shift) to zero. In the ultimate limit of extensive intermixing, the QW becomes a very wide well and energy separation between the eigenstates diminishes, thus $\Delta E_{12}$ has to reduce with longterm interdiffusion. The initial rise also demonstrates a slightly more rapid eigenstate movement for the $E_{2}$ than the $E_{1}$. This is simply because the upper $E_{2}$ state is more weakly bounded and it responds more sensitively to the well shape changes.

It should be noted that although the intersubband transition energy initially blue shifts, the eventual energy red shift with interdiffusion behaves in an opposite way to the conventional interband transition blue shift in energy. One way to explain the two differences is that, for interband transition, after initial interdiffusion has taken place, the gap between the valence and conduction bands enlarges which accounts for a continuous blue shift. In the case of intersubband transitions, it has no direct dependence on the bandgap but only on the well depth, which certainly is reducing with interdiffusion. In other words, intersubband transition is a consequence of the eigenstates separation spacing and interband transition has to account for the bandgap change in the long diffusion time (length) range.

\section{B. Linear and Nonlinear Optical Properties}

It has been found that the higher the densities of electrons inside the GaAs well, the higher the absorption coefficients and the greater the change of refractive indices [10], [21]. This is due to the fact that an increase in electron densities in the QW can enlarge the electron density difference between the lowest two subbands and their respective dipole moment. The absorption coefficients and change of refractive index are shown for both the as-grown and interdiffused structures

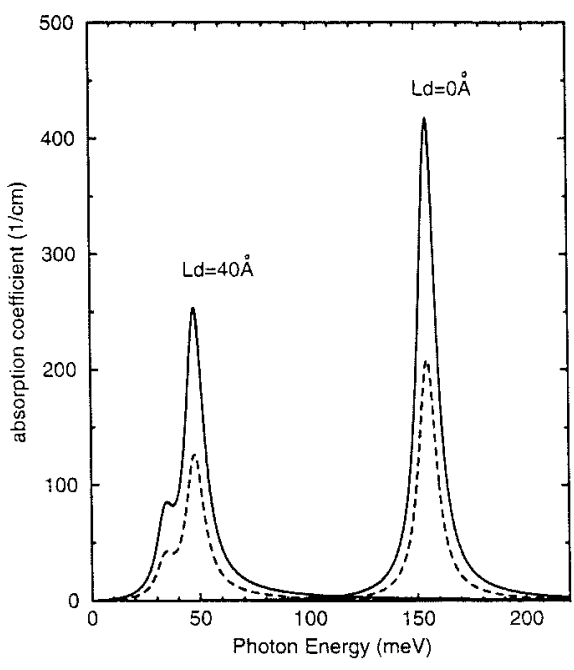

(a)

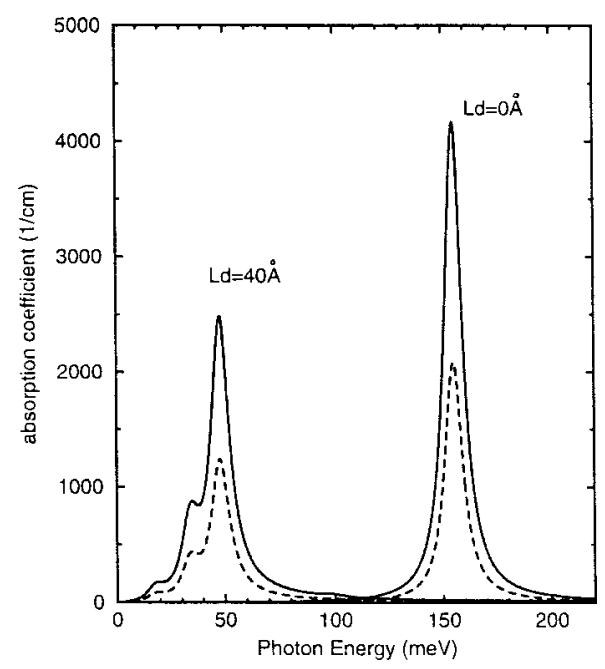

(b)

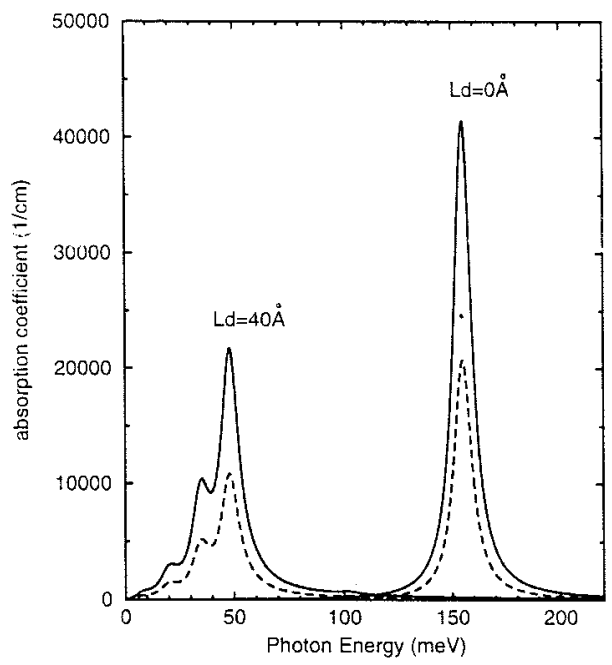

(c)

Fig. 2. (a) The linear and nonlinear intersubband absorption coefficients for as-grown and interdiffused QW with doping concentration $=$ (a) $10^{16}$, (b) $10^{17}$, and (c) $10^{18} \mathrm{~cm}^{-3}$. The solid lines are for cases with incident optical intensity equal to zero and the dashed lines are for the cases with optical intensity equal to saturation intensity. 


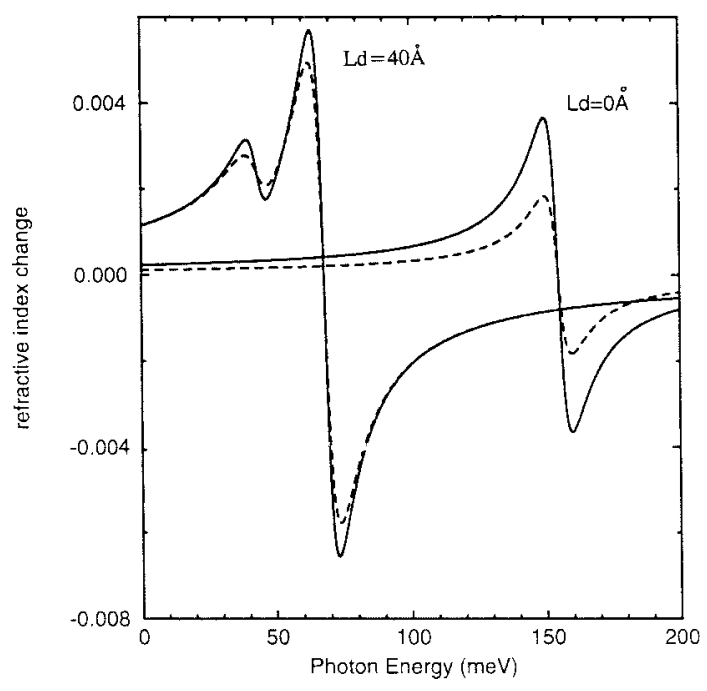

(a)

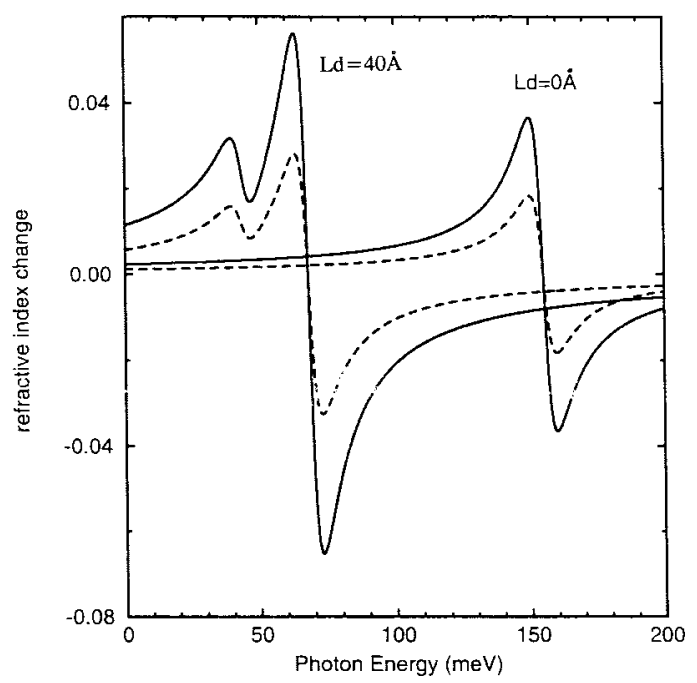

(b)

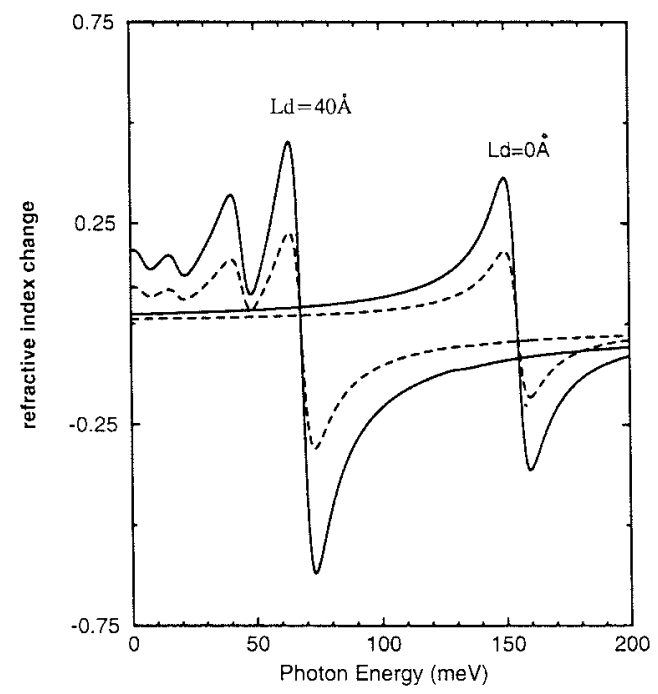

(c)

Fig. 3. (a) The linear and nonlinear intersubband refractive index changes for as-grown and diffused QW with doping concentration $=$ (a) $10^{16}$, (b) $10^{17}$, (c) $10^{18} \mathrm{~cm}^{-3}$. The solid lines are for cases with incident optical intensity equal to zero and the dashed lines are for the cases with optical intensity equal to saturation intensity. The $\mathrm{QW}$ has $\mathrm{Al}$ concentration $=0.3$ and $L_{z}=70 \AA$.

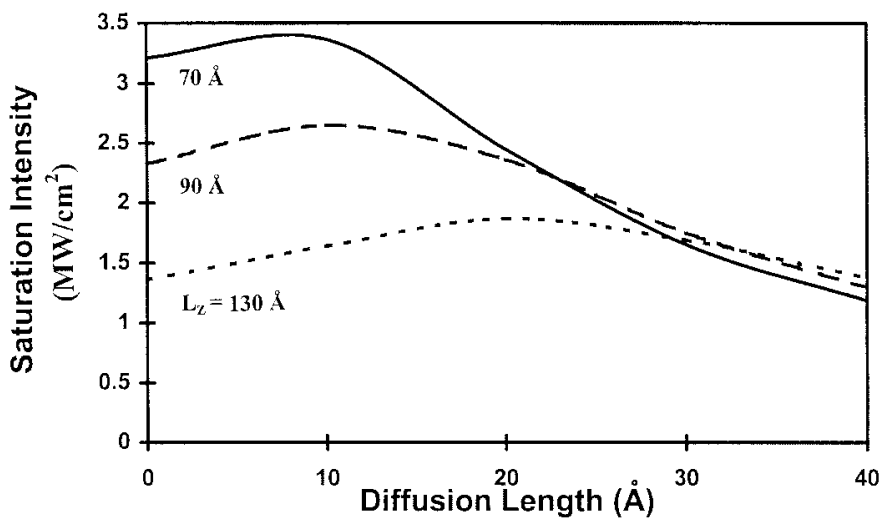

Fig. 4. Saturation intensity versus diffusion length of QW's with different well widths.

in Figs. 2 and 3, respectively. Optical intensity equals zero and intensity set at saturation intensity $I_{\mathrm{op}}^{s}$ are shown for comparison purposes. The absorption coefficients and the refractive index changes are both reduced to half at $I_{\mathrm{op}}^{s}$. For the relatively large $L_{d}$ shown here, fine structures appear at the lower energy end of the interdiffused spectra. These are due to the increasing number of eigenstates pulled down from the continuum to the widened well top for these interdiffusion extents. The effect of injected carriers, as expected, reduces the absorption peak by one order as the carrier's concentration lowers by one order. The values of $I_{\mathrm{op}}^{s}$ as a function of different diffusion lengths are shown in Fig. 4. This value is not sensitive to the level of doping considered here. Its behavior is similar to the subband separation and finally comes to about $1.3 \mathrm{MW} / \mathrm{cm}^{2}$ for all cases. It gives an indication that with the use of optical intensity below this value, the drop of the absorption coefficients will not be too significant. The absorption coefficients generally increase with the doping concentration for all $L_{d}$ ranging up to $40 \AA$. However, in the case of narrow width $L_{z}=70 \AA$, the ratio of absorption drop with increasing interdiffusion is larger for a higher carrier injection level. This may be due to the larger screening effect at higher doping concentration and a drop of the Fermi level for longer diffusion lengths. However, this should require further analysis.

\section{Device Applications}

In the analysis of the DFQW absorption coefficients over a wide range of QW structures, it is found that the fundamental transition energy varies considerably with the diffusion length, and this property can be used for wide-bandwidth photodetection materials. Since the bandwidth of operation increases with the rise of aluminum concentration ratio and the reduction of well width, a wide-bandwidth $(15.9 \mu \mathrm{m}) \mathrm{DFQW}$ material with $L_{z}=70 \AA, w=0.4$, and $L_{d}$ varies from $0 \AA\left(\Delta E_{12}=\right.$ $6.9 \mu \mathrm{m})$ to $40 \AA\left(\Delta E_{12}=22.8 \mu \mathrm{m}\right)$ is proposed here, as shown in Fig. 5. Apart from a wide-bandwidth operation, it can also be used as a material for multiple-color QW infrared photodetectors (QWIP's). As observed in Fig. 5, the overlapping between the adjacent absorption spectrum for different $L_{d}$ 's is so small that a sharp discrepancy for the operation of the multicolor photodetector can be obtained. 


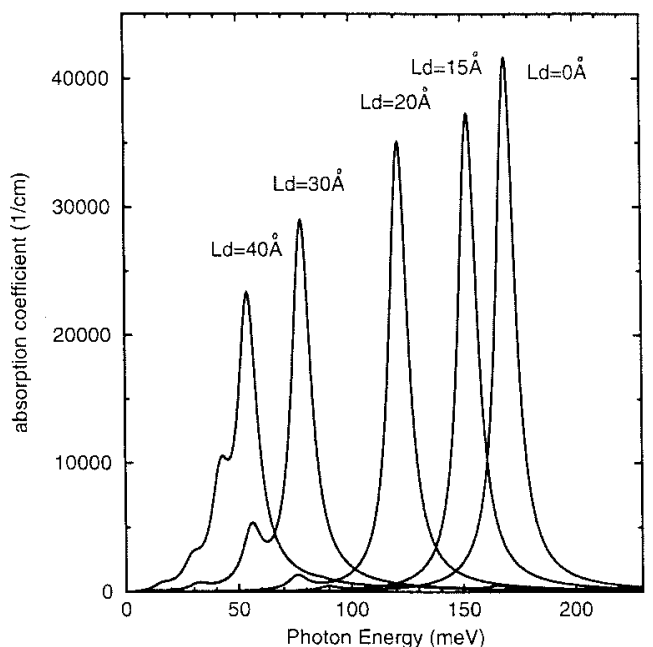

Fig. 5. Absorption coefficients in different diffused QW's for wide-bandwidth photodetection. $L_{z}=70 \AA$ and doping concentration $=10^{17} \mathrm{~cm}^{-3}$.

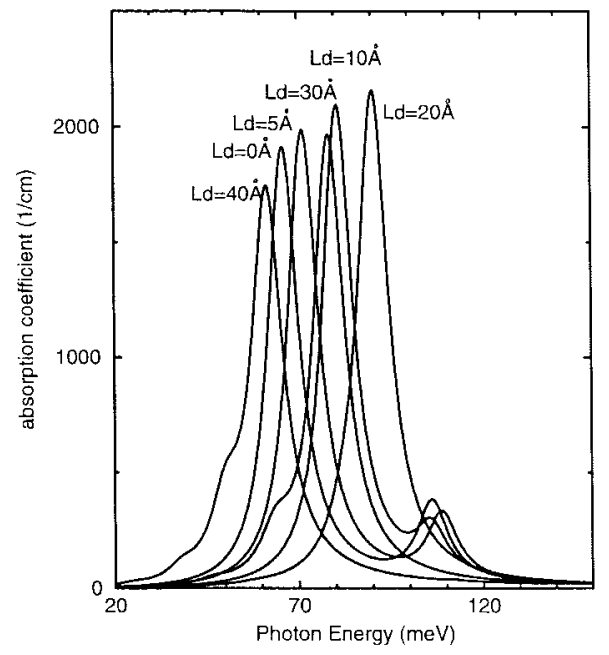

Fig. 6. Absorption coefficients in different diffused QW's for broad-band photodetection. $L_{z}=130 \AA$ and doping concentration $=10^{18} \mathrm{~cm}^{-3}$.

Compared with the two-color photodetector using different well widths [27], the DFQW has an added advantage allowing the fabrication of several wavelengths using the same as-grown size (on one single substrate) with post processing to make them operative at different $L_{d}$ 's. It is therefore attractive from a fabrication and integration point of view. In addition, if we wish to modify the operation wavelength of the QWIP post fabrication, it is fairly flexible if we use the technique of interdiffusion to provide a tunable off-the-shelf material. The low absorption drop as $L_{d}$ increases is also a favorable feature for use as a photodetecting material. In Fig. 5, the operating wavelengths of the optimized DFQW include $6.9 \mu \mathrm{m}\left(L_{d}=\right.$ $0), 7.3 \mu \mathrm{m}\left(L_{d}=10 \AA\right) 10.2 \mu \mathrm{m}\left(L_{d}=20 \AA\right), 16.3 \mu \mathrm{m}$ $\left(L_{d}=30 \AA\right)$ and $22.8 \mu \mathrm{m}\left(L_{d}=40 \AA\right)$, which demonstrates a wide tunability.

A second type of application for the DFQW material for intersubband transitions is observed in DFQW with a larger asgrown width, such as $L_{z}=130 \AA$, shown in Fig. 6 for a broadband operation of $6.5 \mu \mathrm{m}$ wide (13.7-20.2 $\mu \mathrm{m})$. At larger well

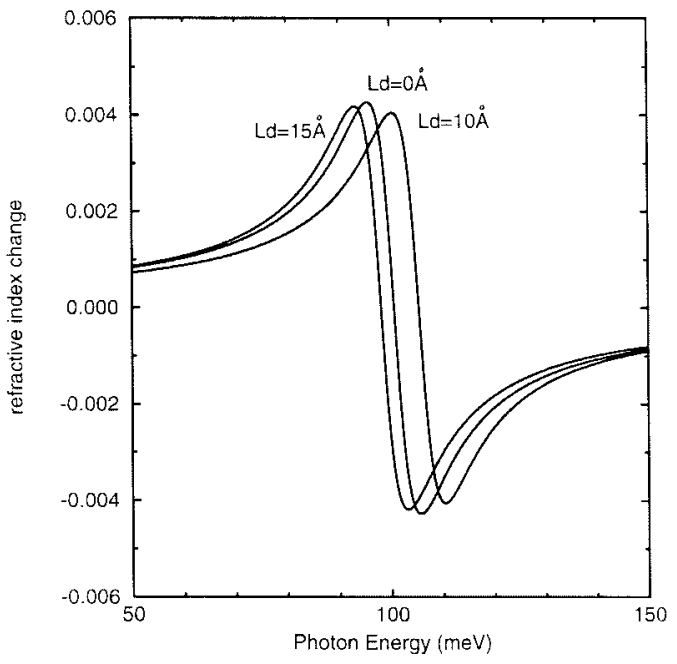

Fig. 7. Variation of the refractive index change spectra for QW's with three diffusion lengths. $L_{z}=90 \AA$, Al concentration $=0.2$ and doping concentration $=10^{16} \mathrm{~cm}^{-3}$.

widths (>100 $\AA$ ), the variations of $L_{d}$ only leads to changes in a small transition energy of the absorption coefficients. However, the absorption peaks remain approximately the same when $L_{d}$ increases (note for smaller well width, e.g., $L_{z}=70$ $\AA$, the peak of absorption reduces as $L_{d}$ is raised). As a result, it is suitable for the design of a broad-band IR photodetector using a DFQW with larger well widths. However, it should be noted that the operation range for $\mathrm{AlGaAs}-\mathrm{GaAs}$ is at a longer IR wavelength region, which is approximately larger than 12 $\mu \mathrm{m}(100 \mathrm{meV})$ for all cases of $\mathrm{Al}$ concentration ratio.

There is a problem with the sensitivity of refractive index change for the QW width set at a predesigned operation wavelength [10]. If the control of the well width $(100 \AA)$ during fabrication at a desired value is more than $10 \AA$, it would cause a large change to the refractive index and also the absorption coefficient. Since it is observed that the change of transition energy is small for a large-well-width DFQW, it can be used to correct the deviation of the transition energies due to the error in well width fabrication. To demonstrate, results on the variation of refractive index change at different $L_{d}$ 's are shown in Fig. 7 for $L_{z}=90 \AA$. It can be seen that the diffusion length from $L_{d}=0$ to $10 \AA$ and $15 \AA$ can shift the transition energies to a range of $10-20 \mathrm{meV}$ [see Fig. 2(c)] with $|\Delta n| \leq$ 0.004 , which is sufficient to compensate the problem of the well width sensitivity.

In the case of a wide as-grown QW (e.g., $L_{z}=130 \AA$ ), there exist minor absorption peaks and dispersions of refractive index change at a higher energy near the fundamental transition, see Figs. 6 and 8. The additional transition appears at the higher energy are due to transition between the lower to upper states while those additional features for cases of large $L_{d}$ (see Figs. 2 and 3) are due to transition between the upper states. The extra transitions for a wider QW width are due to more electron states being bounded. These minor absorption peaks and dispersion of refractive indices are undesirable since they may cause noise or even error in photodetection. However, this problem can be solved by interdiffusion. As shown in Fig. 8, when the diffusion length is increased from 0 to $20 \AA$, the 


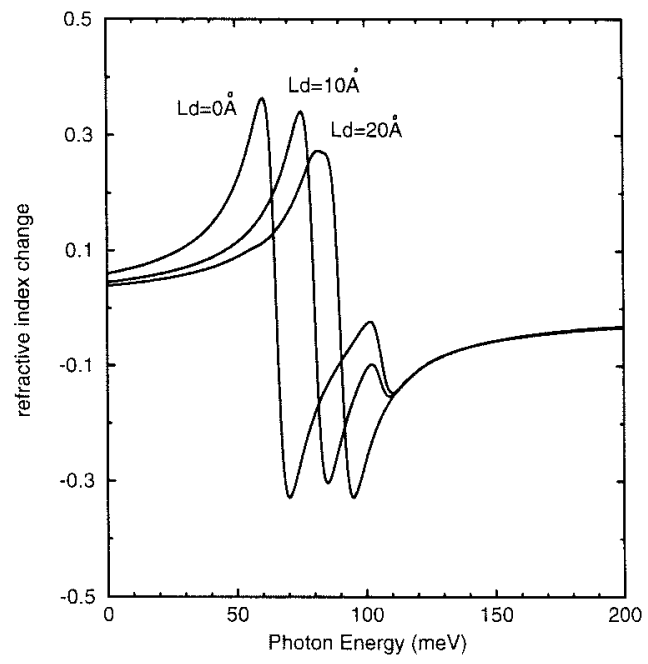

Fig. 8. Refractive index change spectra for QW with three diffusion lengths. $L_{z}=130 \AA$ and doping concentration $=10^{18} \mathrm{~cm}^{-3}$.

dispersion in refractive index change disappears. This is due to the fact that during the initial stage of interdiffusion the QW becomes effectively narrower and hence pushes the states up to result in a clearer transition between $E_{1}$ and $E_{2}$. It should be noted that for a relatively larger diffusion length (e.g., 40 $\AA$ ), minor peaks of absorption coefficients and refractive index changes appear again as the QW width becomes effectively wider.

\section{CONCLUSION}

The linear and nonlinear intersubband absorption and refractive index changes in the interdiffused (intermixed) QW have been studied. Transition energies between the lowest two subbands, optical properties such as the absorption peaks, and the saturation intensities in DFQW's are analyzed in detail. The characteristic of the variation of the transition energies in interdiffused QW with different well widths can be used in application such as the multicolor QW and the broadband photodetector. The shifts in the absorption peak and the refractive index change during the fabrication of the $\mathrm{QW}$ can be corrected by applying a postgrown fine-tuning process using interdiffusion with a relatively small diffusion length. In addition, the technique of QW intermixing can remove (or clear away) the noise generated in the absorption and refractive index change spectra.

The screening effect and the nonlinear effect are taken into account in the calculations. However, the many body effects due to the interactions among the electrons are not considered in this paper. It is important for heavier doping concentrations. More in-depth analysis is required in these areas.

\section{REFERENCES}

[1] M. J. Kelly, Low Dimensional Semiconductors. Oxford, U.K.: Oxford, 1995.

[2] E. H. Li, Ed., Quantum Well Mixing and Optoelectronic Device Applications, Milestone Series. Bellingham WA: SPIE Press, 1998, vol. 145.

[3] T. E. Schlesinger and T. Kuech, "Determination of the interdiffusion of $\mathrm{Al}$ and $\mathrm{Ga}$ in undoped (Al, Ga)As/GaAs quantum well," Appl. Phys. Lett., vol. 49, pp. 519-512, 1986.
[4] Y. Nagai, K. Shigihara, S. Karakida, S. Kakimoto, M. Otsubo, and K. Ikeda, "Characteristics of laser diodes with a partially intermixed GaAs-AlGaAs quantum well," IEEE J. Quantum Electron., vol. 31, pp. 1364-1370, 1995.

[5] L. C. West and S. J. Eglash, "First observation of an extremely large dipole infrared transition within the conduction band of a GaAs quantum well," Appl. Phys Lett., vol. 46, pp. 1156-1158, 1985.

[6] M. O. Manasreh, Ed., Semiconductor Quantum Wells and Superlattices for Long Wavelength Infrared Detectors. Boston, MA: Artech House, 1993.

[7] R. L. Whitney, F. W. Adams, and K. F. Cuff, "Intersubband transitions in quantum wells," in NATO Advanced Research Workshop on Intersubband Transitions in Quantum Wells, NATO ASI Series, Series B: Physics. New York: Plenum, 1992, vol. 188.

[8] A. Kastalsky, "Infrared intraband laser induced in a multiple-quantumwell interband laser," IEEE J. Quantum Electron., vol. 29, pp. 1112-1115, 1993.

[9] E. Rosencher and P. H. Bois, "Model system for optical nonlinearites: Asymmetric quantum wells," Phys. Rev. B, vol. 44, pp. 11315-11327, 1991.

[10] K. J. Kuhn, G. U. Iyengar, and S. Yee, "Free carrier induced change in the absorption and refractive index for intersubband optical transitions in $\mathrm{Al}_{x} \mathrm{Ga}_{1-x} \mathrm{As} / \mathrm{GaAs} / \mathrm{Al}_{x} \mathrm{Ga}_{1-x}$ As quantum wells," J. Appl. Phys., vol. 70, pp. 5010-5017, 1991.

[11] J. D. Ralston, M. Ramsteiner, B. Dischler, M. Maier, G. Brandt, P. Koidl, and D. J. As, "Intersubband transitions in partially interdiffused GaAs/AlGaAs multiple quantum-well structures," J. Appl. Phys., vol. 70, pp. 2195-2199, 1991.

[12] E. H. Li, B. L. Weiss, and A. Laszez, "Nonlinear intersubband optical absorption in diffusion-induced optical $\mathrm{AlGaAs} / \mathrm{GaAs}$ quantum well at far IR wavelengths," Electron. Lett., vol. 28, pp. 885-886, 1992.

[13] A. G. Steele, M. Buchanan, H. C. Liu, and Z. R. Wasilewski, "Postgrowth tuning of quantum well infrared detectors by rapid thermal annealing," J. Appl. Phys., vol. 75, pp. 8234-8236, 1994.

[14] A. Kock, E. Gornik, Abstreiter, G. Bohm, M. Walther, and G. Weimann, "Double wavelength selective GaAs/AlGaAs infrared detector device," Appl. Phys. Lett., vol. 60, pp. 2011-2013, 1992.

[15] A. S. W. Lee and E. H. Li, "Effect of interdiffusion on quantum well IR photodetector," Appl. Phys. Lett., vol. 69, no. 23, pp. 3581-3583, 1996.

[16] S. Y. Yuen, "Fast relaxing absorptive nonlinear refraction in superlattices," Appl. Phys. Lett., vol. 43, pp. 813-815, 1983.

[17] D. Ahn and S. L. Chuang, "Intersubband optical absorption in a quantum well with an applied electric field," Phys. Rev. B, vol. 35, pp. 4149-4151, 1987.

[18] _ "Nonlinear intersubband optical absorption in a semiconductor quantum well," J. Appl. Phys., vol. 62, pp. 3052-3055, 1987.

[19] , "Calculation of linear and nonlinear intersubband optical absorptions in a quantum-well model with an applied electric field," IEEE J. Quantum Electron., vol. QE-23, pp. 2196-2204, 1987.

[20] D. Huang, J. I. Chyi, and H. Morkoc, "Carrier effects on the excitonic absorption in GaAs quantum-well structures: Phase-space filling," Phys. Rev. B., vol. 42, pp. 5147-5153, 1990.

[21] A. Tomita and A. Suzuki, "Carrier-induced lasing wavelength shift for quantum well laser diodes," IEEE J. Quantum Electron., vol. QE-23, pp. 1155-1159, 1987.

[22] E. J. Roan and S. L. Chuang, "Linear and nonlinear intersubband electroabsorptions in a modulation-doped quantum well," J. Appl. Phys., vol. 69, pp. 3249-3260, 1991.

[23] D. J. BenDaniel and C. B. Duke, "Space-charge effects on electron tunneling," Phys. Rev., vol. 152, pp. 683-692, 1966.

[24] E. H. Li. and B. L. Weiss, "Exciton optical absorption in a diffusion induced nonsquare $\mathrm{AlGaAs} / \mathrm{GaAs}$ quantum well," in Quantum Well and Superlattice Physics IV, SPIE Proc., 1992, vol. 1675, pp. 98-108.

[25] D. D. Coon and R. P. G. Karunasiri, "New mode IR detection using quantum wells," Appl. Phys. Lett., vol. 45, pp. 649-651, 1984.

[26] E. H. Li, B. L. Weiss, and K. S. Chan, "Effect of interdiffusion on the subbands in an $\mathrm{Al}_{x} \mathrm{Ga}_{1-x} \mathrm{As} / \mathrm{GaAs}$ single quantum-well structure," Phys. Rev. B, vol. 46, pp. 15181-15192, 1992.

[27] A. S. W. Lee, E. H. Li, and G. Karunasiri, "Impurity free intermixing in InGaAs/GaAs strained multiple quantum well infrared photodetector," in Proc. SPIE-Photodetectors: Materials and Devices III, Conf. 3287-11, San Jose, CA, Jan. 24-30, 1998, vol. 3287, pp. 105-112.

E. Herbert Li (S'87-M'88-SM'95), for photograph and biography, see p. 990 of the June 1998 issue of this JournaL. 